\title{
Atomic Structure of Self-Pillared, Single-Unit-Cell Sn-MFI Zeolite Nanosheets
}

\author{
Prashant Kumar, ${ }^{1}$ Limin Ren, ${ }^{1}$ Qiang Guo, ${ }^{1}$ Xueyi Zhang, ${ }^{2}$ Michael Tsapatsis, ${ }^{1}$ K. Andre Mkhoyan ${ }^{1}$ \\ ${ }^{1}$ Department of Chemical Engineering \& Materials Science, University of Minnesota, Minneapolis, MN 55455. \\ ${ }^{2}$ Department of Chemical Engineering, The Pennsylvania State University, University Park, PA 16802.
}

Two-dimensional MFI-type zeolites are single or near single-unit-cell thick microporous nanosheets with pore sizes of $\sim 5 \AA$ in diameter, formed by a network of interconnected silicon and oxygen atoms [1-3]. These nanosheets can be fabricated into ultra-thin membranes for the separation of isomer mixtures, such as p-xylene/o-xylene and n-butane/i-butane by allowing only the passage of isomer that fits within its micropores. In addition, application of these nanosheets can be further extended by incorporating heteroatoms, such as Sn atoms, into its framework as catalytically active sites for the isomerization of glucose to fructose, the reaction involved in production of high-fructose corn syrup. The yield of fructose during this reaction depends on the accessibility of catalytically active sites in the zeolite framework.

In this work, bright-field transmission electron microscopy (BF-TEM) is used to study the threedimensional hierarchical arrangement of criss-crossing single-unit-cell thick MFI nanosheets [4]. It is shown through BF-TEM that these hierarchical structures of $\sim 100 \mathrm{~nm}$ in diameter are composed of $90^{\circ}$ intergrowth of nanosheets. High-resolution conventional TEM (HR-TEM) images along [100], [010] and [001] direction reveal that these nanosheets are of MFI-type zeolite framework, with thickness of 2 $\mathrm{nm}$ along [010] direction (Figure 1a-f). HR-TEM images acquired at near Scherzer defocus conditions from bulk MFI crystals further corroborate the atomic structure of these nanosheets (Figure 1g-1). FEI Tecnai G2 F30 (S)TEM equipped with TWIN pole piece and a Schottky field emission electron gun operating at $300 \mathrm{kV}$ was used for this analysis.

Furthermore, a comparative study was carried out on three different $\mathrm{Sn}$ incorporated MFI specimens with $\mathrm{Si} / \mathrm{Sn}$ atomic ratios of 75, 186, and 223 using high-angle annular dark-field scanning transmission electron microscopy (HAADF-STEM) imaging. Aberration corrected FEI Titan 60-300 (S)TEM, equipped with FEI SuperX EDX detector, operating at $300 \mathrm{kV}$ was used in these measurements. Distribution of such minute amount of $\mathrm{Sn}$ atoms/clusters was mapped by analyzing the energy dispersive X-ray signal (EDX) acquired at low-dose STEM (Figure 2) operational conditions to minimize the electron beam damage. It is concluded that $\mathrm{Sn}$ atoms form clusters of $\sim 5 \mathrm{~nm}$ size when $\mathrm{Si} / \mathrm{Sn}$ atomic ratio is 75 and 186, and are incorporated in the framework as single atoms when $\mathrm{Si} / \mathrm{Sn}$ atomic ratio is 223 [5]. This correlated with the best catalytic performance reported up to now for sugar isomerization in a heterogeneous catalyst, thus providing a link between the observed catalytic performance of this novel catalyst and the structure and location of the Sn (Lewis-acid) active sites responsible for the catalytic activity [6].

\section{References:}

[1] M. Choi et al, Nature 461, (2009), 246-249.

[2] P. Kumar et al, Nat. Commun. 6, (2015), 7128.

[3] K. Varoon et al, Science 334, (2011), 72-75.

[4] X. Zhang et al, Science 336, (2012), 1684-1687.

[5] L. Ren et al, Angew. Chem. Int. Ed. 54, (2015), 10848-10851. 
[6] This work was supported as part of the Catalysis Center for Energy Innovation, an Energy Frontier Research Center funded by the U.S. Department of Energy, Office of Science, Basic Energy Sciences under Award DE-SC0001004.
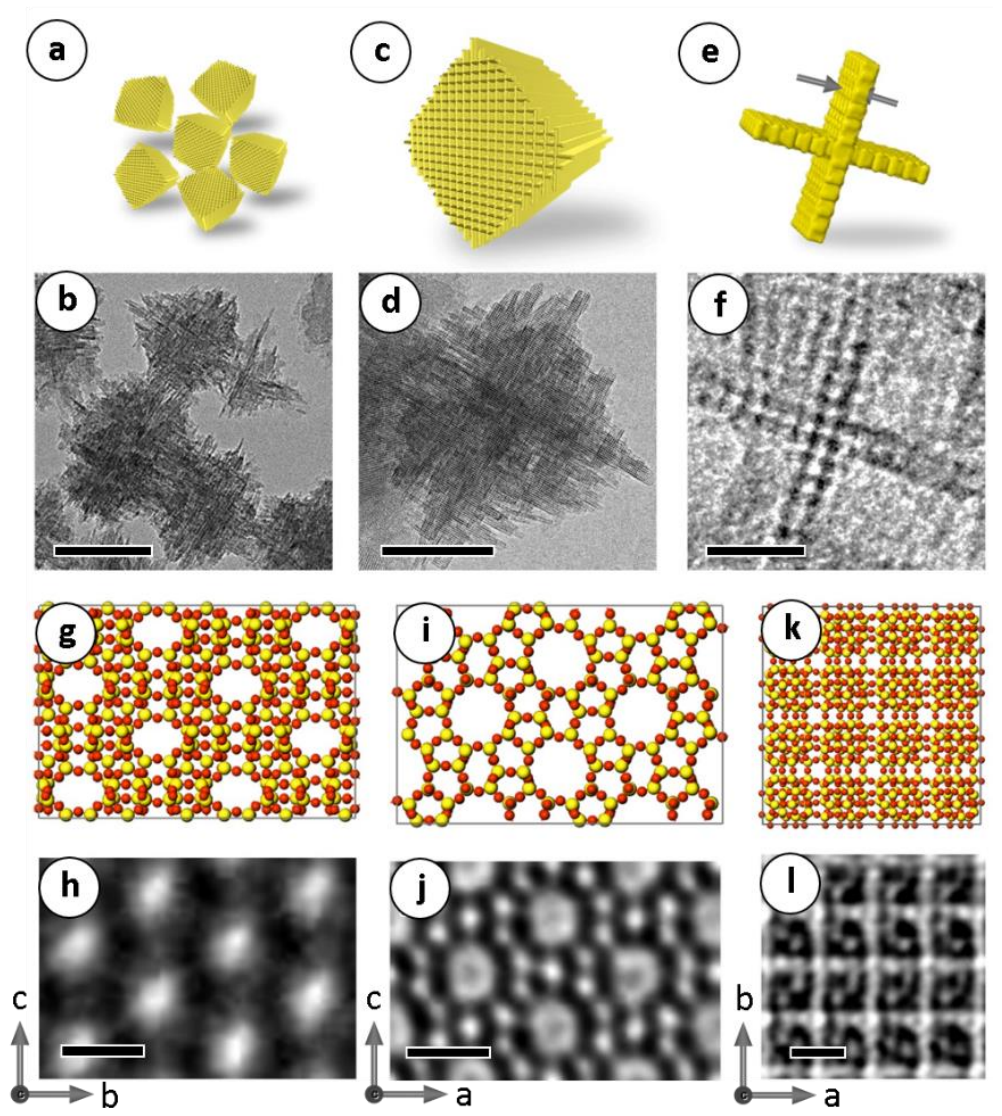

Figure 1. High-resolution TEM images of hierarchical MFI-type zeolite structure. (a),(b) Low magnification image of several particles of $\sim 100 \mathrm{~nm}$ in diameter. (c),(d) Schematic and BF-TEM image of a single particle showing criss-cross arrangement of several nanosheets. (e),(f) Schematic and HR-TEM image of a $90^{\circ}$ intergrowth of two single-unit-cell thick MFI-type zeolite nanosheets. Crystal structure and filtered atomic resolution HR-TEM image of a 2x2x2 supercell of MFItype zeolite along (g),(h) [100] zone axis, (i),(j) [010] zone axis and (k),(l) [001] zone axis. Silicon atoms are shown in yellow and oxygen atoms are shown in red. Scale bar in (b) is $100 \mathrm{~nm}$, (d) is $50 \mathrm{~nm}$, (f) is $5 \mathrm{~nm}$, (h),(j),(l) is $1 \mathrm{~nm}$.
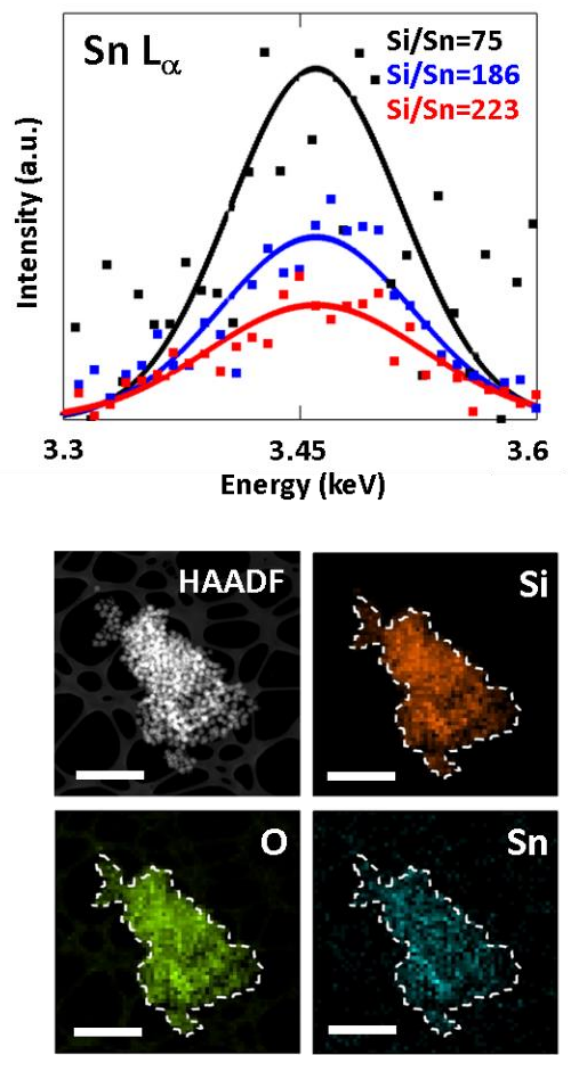

Figure 2. HAADF-STEM EDX mapping of $\mathrm{Sn}$ incorporated hierarchical MFI zeolite. (Top) Background subtracted Sn L $\alpha$ peak acquired from an area averaged EDX spectrum of a cluster of particles with atomic ratios of $\mathrm{Si} / \mathrm{Sn}=75,186,223$. Solid lines represent fitted curves to the experimental scatter data shown. $\mathrm{Sn} \mathrm{L}_{\alpha}$ peak is normalized to $\mathrm{Si}-\mathrm{K}$ peak for comparison. (Bottom) Spatial maps showing uniform distribution of $\mathrm{Sn}$ in a cluster of MFI particles. Scale bars are $2 \mu \mathrm{m}$. 